\title{
Implémentation éléments finis du modèle hyperélastique anisotrope HGO
}

\author{
François Peyraut $^{1}$ - Dominique Chamoret ${ }^{1}-$ Samuel Gomes $^{1}$ \\ Zhi-Qiang Feng ${ }^{2}$
}

1. Laboratoire M3M, Université de Technologie de Belfort Montbéliard

F-90010 Belfort cedex

\{francois.peyraut,dominique.chamoret, samuel,gomes\}@utbm.fr

2. Laboratoire de Mécanique d'Evry - Université d'Evry

40 Rue du Pelvoux, F-91020 Evry

\{Zhi-Qiang.Feng\}@iup.univ-evry.fr

RÉSUMÉ. Les lois de comportements hyperélastiques anisotropes sont souvent utilisées pour déterminer les déformations et les contraintes au sein des tissus biologiques comme les ligaments, les tendons ou les parois artérielles. Dans cet article, on présente l'implémentation éléments finis du modèle HGO dans le code de calcul FER. Trois exemples numériques sont étudiés : traction uniaxiale homogène pour laquelle une solution analytique est disponible; traction uniaxiale simple soulignant le caractère non isotrope de la loi de comportement (rétrécissement et gonflement de la section de l'échantillon dans deux directions perpendiculaires) ; contact et impact main-tissu biologique dans le cadre d'une application avec un mannequin virtuel.

ABSTRACT. Anisotropic hyperelastic constitutive laws are often used to determine strain and stress in biological soft tissues such as ligaments, tendons or arterial walls. In this paper, the implementation of the HGO model in the finite element code FER is presented. Three numerical examples are studied: homogeneous uniaxial tension test where analytical solutions are available; uniaxial tension test highlighting the anisotropic behavior (contracting and swelling of the section in two perpendicular directions); contact and impact between hand and soft biological tissues in the framework of application using a virtual mannequin generator.

MOTS-CLÉS: biomécanique, hyperélasticité anisotrope, modèle HGO, éléments finis.

KEYWORDS: biomechanics, anisotropic hyperelasticity, HGO model, finite element.

DOI:10.3166/EJCM.19.441-464 @ 2010 Lavoisier, Paris 


\section{Introduction}

Pour déterminer les déformations et les contraintes au sein de tissus biologiques tels que les ligaments, les tendons ou les parois artérielles, les lois de comportements hyperélastiques anisotropes sont souvent utilisées dans le cadre de la méthode des éléments finis (Weiss et al., 1996). Les densités d'énergie les plus utilisées impliquent des lois puissance ou exponentielles (Fung et al., 1979 ; Holzapfel et al., 2000 ; Schröder et al., 2005). Comme il est admis que l'anisotropie est due aux fibres de collagène (Gasser et al., 2006), alors que la matrice se comporte de manière isotrope, la densité d'énergie comporte généralement une partie isotrope et une partie anisotrope (Weiss et al., 1996). Chaque densité anisotrope fait référence à une famille de fibres représentant une direction privilégiée du matériau. Dans la loi HGO proposée par (Holzapfel et al., 2000), deux densités anisotropes, correspondant à deux familles de fibres distinctes, sont superposées. Dans cet article, on présente l'implémentation éléments finis du modèle HGO dans le code de calcul FER (FER, 2009). L'implémentation a été réalisée en utilisant une approche lagrangienne totale (Bathe, 1982). Dans les applications nécessitant une interaction avec le milieu environnant, le contact a été traité par la méthode du bi-potentiel (De Saxcé et al., 1991, 1998).

Trois exemples numériques ont été étudiés. Le premier concerne l'essai de traction uniaxiale homogène utilisé pour identifier les propriétés mécaniques de bandes de tissus artériels (Holzapfel, 2006 ; Balzani et al., 2006). Les déformations associées à cet essai étant homogènes, il est possible de calculer analytiquement la solution. Grâce à un changement d'inconnue approprié, les équations d'équilibre se réduisent à une équation polynômiale de degré 3 que l'on résout algébriquement par les formules de Cardan (Peyraut et al., 2009). Les résultats numériques montrent une parfaite concordance avec la solution analytique. On retrouve en particulier la valeur de l'angle critique entre les fibres de collagène et la direction circonférentielle (Guo et al., 2006).

Le deuxième exemple traite de la traction uniaxiale simple. Les déformations ont cette fois-ci un caractère non homogène. Les résultats sont similaires à ceux obtenus dans (Gasser et al., 2006) et soulignent le caractère non isotrope de la loi de comportement. On observe en effet un rétrécissement de la section de l'échantillon dans une direction et un gonflement dans la direction perpendiculaire, ce qui est typique d'un comportement anisotrope.

Le troisième exemple porte sur le contact entre une main et un tissu biologique afin de modéliser l'interaction de la main avec le milieu qui l'environne. Cet exemple est représentatif de situations rencontrées dans les simulateurs chirurgicaux de gestes opératoires, dans lesquels des parties du corps humain, comme les mains $\mathrm{du}$ praticien, interagissent avec des objets rigides, comme les instruments chirurgicaux, ou avec des tissus biologiques représentant les organes du patient virtuel (Talbi, 2008). Pour cet exemple, la représentation géométrique de la main a été extraite du mannequin virtuel MANERCOS (Gomes et al., 1999). 


\section{Hyperélasticité anisotrope}

Les densités d'énergie les plus utilisées en hyperélasticité anisotrope impliquent des lois puissance (Schröder et al., 2005) ou des lois exponentielles (Fung et al., 1979 ; Holzapfel et al., 2000). Il est généralement admis que l'anisotropie est due aux fibres de collagène (Gasser et al., 2006) alors que la matrice se comporte de manière isotrope. C'est la raison pour laquelle la densité d'énergie $W$ est séparée en une partie isotrope et une partie anisotrope (Weiss et al., 1996) :

$$
W=W_{i s o}+\sum_{a=1}^{n} W_{a n i}^{a}
$$

Chaque densité anisotrope $W_{a n i}^{a}$ fait référence à une direction privilégiée $\mathrm{du}$ matériau. Pour modéliser les ligaments ou les tendons, le nombre de famille de fibres $n$ est égal à 1 . Il est égal à 2 pour représenter le comportement des parois artérielles. Dans la loi HGO proposée par (Holzapfel et al., 2000), deux densités anisotropes sont superposées avec deux directions privilégiées $\mathbf{a}^{1}$ et $\mathbf{a}^{2}$ correspondant à deux familles de fibres distinctes :

$$
\mathbf{a}^{1}=\{\cos (\beta), \sin (\beta), 0\}^{T} ; \quad \mathbf{a}^{2}=\{\cos (\beta),-\sin (\beta), 0\}^{T}
$$

où $\beta$ représente l'angle entre les fibres de collagène et la direction circonférentielle sur des tissus extraits d'aorte abdominale humaine (figure 1). Dans la suite, on notera en abrégé $c, s$ et $t$ le cosinus, le sinus et la tangente de l'angle $\beta$.

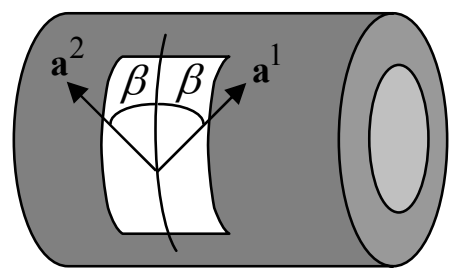

Figure 1. Angle $\beta$

Les lois hyperélastiques anisotropes incluent les trois invariants classiques $I_{1}, I_{2}$ et $I_{3}$ de la matrice de dilation $\mathbf{C}$ ainsi que deux invariants additionnels $J_{4}^{a}$ et $J_{5}^{a}$ liés à l'anisotropie des tissus et définis en fonction du tenseur structurel $\mathbf{M}^{\mathrm{a}}$ (Spencer, 1987) :

$$
I_{1}=\operatorname{tr}(\mathbf{C}), I_{2}=\operatorname{tr}(\operatorname{cof}(\mathbf{C})), I_{3}=\operatorname{det}(\mathbf{C}), J_{4}^{a}=\operatorname{tr}\left(\mathbf{C} \mathbf{M}^{\mathrm{a}}\right), J_{5}^{a}=\operatorname{tr}\left(\mathbf{C}^{2} \mathbf{M}^{\mathrm{a}}\right)[3]
$$

Le tenseur structurel $\mathbf{M}^{\mathrm{a}}$, qui représente l'isotropie transverse, est relié à la direction préférentielle des fibres $\mathbf{a}$ : 


$$
\left(\mathbf{M}^{\mathrm{a}}\right)_{\mathrm{ij}}=(\mathbf{a} \otimes \mathbf{a})_{\mathrm{ij}}=\mathrm{a}_{\mathrm{i}} \mathrm{a}_{\mathrm{j}}
$$

Dans le cas du modèle HGO, ce tenseur s'écrit, en tenant compte de [2] :

$$
\mathbf{M}^{\mathbf{a}^{1}}=\left[\begin{array}{ccc}
c^{2} & c s & 0 \\
c s & s^{2} & 0 \\
0 & 0 & 0
\end{array}\right] \quad ; \quad \mathbf{M}^{\mathbf{a}^{2}}=\left[\begin{array}{ccc}
c^{2} & -c s & 0 \\
-c s & s^{2} & 0 \\
0 & 0 & 0
\end{array}\right]
$$

Classiquement, le second tenseur des contraintes de Piola-Kirchhoff $\mathbf{S}$ dérive de la densité $W$ :

$$
\mathbf{S}=\frac{\partial W}{\partial \mathbf{E}}=2 \frac{\partial W}{\partial \mathbf{C}}
$$

où $\mathbf{E}$ et $\mathbf{C}$ représentent respectivement les tenseurs de déformations de GreenLagrange et de Cauchy-Green à droite :

$$
\mathbf{E}=\frac{1}{2}(\mathbf{C}-\mathbf{I}) ; \mathbf{C}=\mathbf{F}^{\mathrm{T}} \mathbf{F} ; \mathbf{F}=\mathbf{I}+\frac{\partial \mathbf{u}}{\partial \mathbf{X}}
$$

$\mathbf{F}$ étant le gradient de la transformation, $\mathbf{u}$ le déplacement, $\mathbf{I}$ le tenseur unité et $\mathbf{X}$ les coordonnées lagrangiennes. Afin de distinguer la partie déviatorique des contraintes de la partie sphérique, qui correspond à une dilatation pure, il est classique d'utiliser la décomposition suivante (Weiss et al., 1996) :

$$
\mathbf{F}=\mathbf{F}_{v o l} \overline{\mathbf{F}} ; \mathbf{F}_{v o l}=J^{1 / 3} \mathbf{I} ; \overline{\mathbf{F}}=J^{-1 / 3} \mathbf{F}
$$

où $J$ représente le déterminant de la matrice jacobienne de la déformation :

$$
J=\operatorname{det}(\mathbf{F})
$$

On a alors de manière évidente :

$$
\operatorname{det}\left(\mathbf{F}_{v o l}\right)=J ; \operatorname{det}(\overline{\mathbf{F}})=1
$$

Le terme $\mathbf{F}_{v o l}$ contribue donc seul au changement de volume. On en déduit la décomposition suivante pour le tenseur des déformations de Cauchy-Green à droite :

$$
\overline{\mathbf{C}}=\overline{\mathbf{F}}^{T} \overline{\mathbf{F}}=J^{-2 / 3} \mathbf{C}
$$

Les invariants réduits associés à $\overline{\mathbf{C}}$ se déduisent de ceux de $\mathbf{C}$ par :

$$
\bar{I}_{1}=I_{1} I_{3}^{-1 / 3} ; \bar{I}_{2}=I_{2} I_{3}^{-2 / 3} ; \bar{J}_{4}^{a}=J_{4}^{a} I_{3}^{-1 / 3} ; \bar{J}_{5}^{a}=J_{5}^{a} I_{3}^{-2 / 3}
$$


Le modèle HGO utilise la formulation en invariants réduits avec la densité d'énergie $W$ définie par :

$$
\begin{aligned}
& W=\bar{W}\left(\bar{I}_{1}, \bar{J}_{4}^{a}\right)+U(J) \\
& U(J)=\frac{k}{2}(J-1)^{2} \\
& \bar{W}\left(\bar{I}_{1}, \bar{J}_{4}^{a}\right)=W_{\text {iso }}\left(\bar{I}_{1}\right)+\sum_{a=1}^{2} W_{\text {ani }}\left(\bar{J}_{4}^{a}\right) \\
& W_{\text {iso }}\left(\bar{I}_{1}\right)=c_{1}\left(\bar{I}_{1}-3\right) \\
& J_{4}^{a}<1: W_{\text {ani }}\left(\bar{J}_{4}^{a}\right)=0 \\
& J_{4}^{a} \geq 1: W_{\text {ani }}\left(\bar{J}_{4}^{a}\right)=\frac{k_{1}}{2 k_{2}}\left[\exp \left(k_{2}\left(\bar{J}_{4}^{a}-1\right)^{2}\right)-1\right]
\end{aligned}
$$

Le terme $\bar{W}\left(\bar{I}_{1}, \bar{J}_{4}^{a}\right)$ représente la composante déviatorique qui est découplée du terme de dilatation représenté par $U(J)$. La contribution isotrope $W_{i s o}$ est de type néohookéen alors que la contribution anisotrope $W_{a n i}$ est de type exponentiel. Cette dernière ne dépend que du quatrième invariant. Comme $J_{4}^{a}$ représente le carré de l'allongement dans la direction a, et que la densité est nulle lorsque $J_{4}^{a}$ est plus petit que 1, le modèle HGO prend en compte le fait que les fibres ne travaillent pas en compression. Les paramètres matériels $c_{1}, k_{1}$ et $k_{2}$ ont été choisis comme dans (Balzani et al., 2006), de manière à ajuster le modèle avec des données expérimentales : $c_{1}=10.2069 \mathrm{kPa} ; k_{1}=0.0017 \mathrm{kPa} ; k_{2}=882.847$. On supposera que ces paramètres ne dépendent pas de la direction a des fibres, définie par [2], ce qui est légitime car les propriétés des fibres sont indépendantes de leur orientation. En pratique, le terme de dilatation $U(J)$ peut être considéré comme une contrainte de pénalité, inclus dans le modèle éléments finis pour tenir compte de l'incompressibilité du matériau. Le paramètre $k$ a été choisi égal à $10^{5}$. On rappelle enfin, qu'en dérivant $W$ à partir de [6] et en introduisant la matrice des cofacteurs de $\mathbf{C}, \operatorname{Cof}(\mathbf{C})=\operatorname{det}(\mathbf{C}) \mathbf{C}^{-\mathrm{T}}$, on obtient classiquement (Schröder et al., 2005) :

$$
\begin{aligned}
& \mathbf{S}=2\left[\frac{\partial W}{\partial I_{1}} \mathbf{I}+\frac{\partial W}{\partial I_{2}}\left(I_{1} \mathbf{I}-\mathbf{C}\right)+\frac{\partial W}{\partial I_{3}} \operatorname{cof}(\mathbf{C})+\frac{\partial W}{\partial J_{4}^{1}} \mathbf{M}^{\mathbf{a}^{1}}+\frac{\partial W}{\partial J_{4}^{2}} \mathbf{M}^{\mathbf{a}^{2}}\right. \\
& \left.+\frac{\partial W}{\partial J_{5}^{1}}\left(\mathbf{C} \mathbf{M}^{\mathbf{a}^{1}}+\mathbf{M}^{\mathbf{a}} \mathbf{C}\right)+\frac{\partial W}{\partial J_{5}^{2}}\left(\mathbf{C} \mathbf{M}^{\mathbf{a}^{2}}+\mathbf{M}^{\mathbf{a}^{2}} \mathbf{C}\right)\right]
\end{aligned}
$$


Pour le cas particulier qui nous intéresse, l'expression générale [19] se réduit à :

$$
\mathbf{S}=2\left[\frac{\partial W}{\partial I_{1}} \mathbf{I}+\frac{\partial W}{\partial I_{3}} \operatorname{cof}(\mathbf{C})+\frac{\partial W}{\partial J_{4}^{1}} \mathbf{M}^{\mathbf{a}^{1}}+\frac{\partial W}{\partial J_{4}^{2}} \mathbf{M}^{\mathbf{a}^{2}}\right]
$$

où les dérivées premières de la densité d'énergie par rapport aux invariants se calculent à partir de [13]-[18] :

$$
\begin{aligned}
& \frac{\partial W}{\partial I_{1}}=c_{1} I_{3}^{-1 / 3} \\
& \frac{\partial W}{\partial I_{3}}=-\frac{1}{3 I_{3}}\left[c_{1} \bar{I}_{1}+\sum_{a=1}^{2} \bar{J}_{4}^{a} \frac{d W_{a n i}}{d \bar{J}_{4}^{a}}\right]+\frac{U^{\prime}(J)}{2 J} \\
& \frac{\partial W}{\partial J_{4}^{a}}=I_{3}-1 / 3 \frac{d W_{\text {ani }}}{d \bar{J}_{4}^{a}} \\
& U^{\prime}(J)=k(J-1) \\
& \frac{d W_{\text {ani }}}{d \bar{J}_{4}^{a}}=k_{1}\left(\bar{J}_{4}^{a}-1\right) \exp \left(k_{2}\left(\bar{J}_{4}^{a}-1\right)^{2}\right) H\left(J_{4}^{a}\right)
\end{aligned}
$$

La fonction de Heaviside $H$ a été introduite dans [25] pour prendre en compte le fait que la contribution anisotrope de la densité HGO est nulle lorsque le matériau est en compression dans la direction des fibres.

Les contraintes de Cauchy $₫$ se déduisent de $\mathbf{S}$ par la relation classique :

$$
\sigma=\frac{1}{J} \mathbf{F} \mathbf{S} \mathbf{F}^{T}
$$

Afin de mettre en évidence la séparation entre la partie sphérique et la partie déviatorique des contraintes, on introduit l'opérateur déviatorique dev (Weiss et al., 1996) :

$$
\theta=U^{\prime}(J) \mathbf{I}+\frac{2}{J} \operatorname{dev}\left[\overline{\mathbf{F}} \frac{\partial \bar{W}}{\partial \overline{\mathbf{C}}} \overline{\mathbf{F}}^{T}\right] ; \operatorname{dev}[]=[]-\frac{1}{3}(\operatorname{trace}[]) \mathbf{I}
$$

Le premier terme de [27] correspond à la partie sphérique des contraintes, associée à la dilatation, et le second terme correspond à la partie déviatorique. En utilisant [8], [11] et [15]-[18], il résulte finalement de [27] que : 


$$
\begin{aligned}
& g=U^{\prime}(J) \mathbf{I}+2 J^{-5 / 3}\left[c_{1}\left(\mathbf{B}-\frac{I_{1}}{3} \mathbf{I}\right)+\frac{d W_{\text {ani }}}{d \bar{J}_{4}^{1}}\left(\mathbf{F} \mathbf{a}^{1} \otimes \mathbf{F} \mathbf{a}^{1}-\frac{\bar{J}_{4}^{1}}{3} \mathbf{I}\right)\right. \\
& \left.+\frac{d W_{\text {ani }}}{d \bar{J}_{4}^{2}}\left(\mathbf{F} \mathbf{a}^{2} \otimes \mathbf{F} \mathbf{a}^{2}-\frac{\bar{J}_{4}^{2}}{3} \mathbf{I}\right)\right]
\end{aligned}
$$

où $\mathbf{B}=\mathbf{F F}^{\mathrm{T}}$ représente le tenseur des déformations de Cauchy-Green à gauche. Les contraintes [20], ainsi que les dérivées premières [21]-[25], seront utilisées pour le calcul des modules tangents. Ces modules sont nécessaires à l'implémentation des modèles hyperélastiques dans une formulation éléments finis lagrangienne totale. L'implémentation du modèle HGO fait l'objet de la section 3.

\section{Implémentation éléments finis du modèle HGO}

Le modèle HGO a été implémenté dans le code de calcul aux éléments finis FER en formulation lagrangienne totale (Bathe, 1982). La programmation a été réalisée en $\mathrm{C}++$, avec une approche orientée objet (Feng et al., 2002). En raison des grands déplacements et des grandes rotations hyperélastiques, le tenseur des déformations de Green-Lagrange $\mathbf{E}$ est adopté pour décrire la relation non linéaire entre les déformations et les déplacements nodaux $\mathbf{u}$ :

$$
\mathbf{E}=\left(\mathbf{B}_{\mathrm{L}}+\frac{1}{2} \mathbf{B}_{\mathrm{NL}}(\mathbf{u})\right) \mathbf{u}
$$

où $\mathbf{B}_{\mathrm{L}}$ et $\mathbf{B}_{\mathrm{NL}}(\mathbf{u})$ sont les matrices qui relient respectivement la partie linéaire et la partie non linéaire des déformations aux déplacements nodaux. On en déduit la forme incrémentale de la relation entre les déformations et les déplacements :

$$
\delta \mathbf{E}=\left(\mathbf{B}_{\mathrm{L}}+\mathbf{B}_{\mathrm{NL}}(\mathbf{u})\right) \delta \mathbf{u}
$$

Il résulte par ailleurs de [6] et [30] que l'incrément de contrainte $\delta \mathbf{S}$ s'exprime en fonction de l'incrément de déformations $\delta \mathbf{E}$ par :

$$
\delta \mathbf{S}=\frac{\partial^{2} W}{\partial \mathbf{E}^{2}}: \delta \mathbf{E}=\mathbf{D}: \delta \mathbf{E}=\mathbf{D}:\left(\mathbf{B}_{\mathrm{L}}+\mathbf{B}_{\mathrm{NL}}(\mathbf{u})\right) \delta \mathbf{u}
$$

Les modules tangents D utilisés dans la linéarisation [31] de la loi de comportement s'expriment classiquement à l'aide des dérivées premières et secondes de la densité d'énergie par (Schröder et al., 2005) : 


$$
\begin{aligned}
& D_{i j k l}=4\left[\frac{\partial^{2} W}{\partial I_{1} \partial I_{3}}\left(\delta_{i j} \operatorname{Cof}(\mathbf{C})_{k l}+\delta_{k l} \operatorname{Cof}(\mathbf{C})_{i j}\right)\right. \\
& +\frac{\partial^{2} W}{\partial I_{3} \partial I_{3}}\left(\operatorname{Cof}(\mathbf{C})_{i j} \operatorname{Cof}(\mathbf{C})_{k l}\right)+\frac{\partial W}{\partial I_{3}} I_{3}\left(\mathbf{C}_{i j}^{-1} \mathbf{C}_{k l}^{-1}-\mathbf{C}_{i k}^{-1} \mathbf{C}_{j l}^{-1}\right) \\
& +\frac{\partial^{2} W}{\partial J_{4}^{1} \partial J_{4}^{1}} M_{i j}^{\mathbf{a}^{1}} M_{k l}^{\mathbf{a}^{1}}+\frac{\partial^{2} W}{\partial J_{4}^{2} \partial J_{4}^{2}} M_{i j}^{\mathbf{a}^{2}} M_{k l}^{\mathbf{a}^{2}} \\
& +\frac{\partial^{2} W}{\partial I_{3} \partial J_{4}^{1}}\left(\operatorname{Cof}(\mathbf{C})_{i j} M_{k l}^{\mathbf{a}^{1}}+M_{i j}^{\mathbf{a}} \operatorname{Cof}(\mathbf{C})_{k l}\right) \\
& \left.+\frac{\partial^{2} W}{\partial I_{3} \partial J_{4}^{2}}\left(\operatorname{Cof}(\mathbf{C})_{i j} M_{k l}^{\mathbf{a}^{2}}+M_{i j}^{\mathbf{a}^{2}} \operatorname{Cof}(\mathbf{C})_{k l}\right)\right]
\end{aligned}
$$

Les dérivées secondes de $W$ se calculent à partir de [21]-[25] :

$$
\begin{aligned}
& \frac{\partial^{2} W}{\partial I_{1} \partial_{3}}=-\frac{1}{3} c_{1} I_{3}^{-4 / 3} \\
& \frac{\partial^{2} W}{\partial I_{3} \partial I_{3}}=\frac{4\left[c_{1} \bar{I}_{1}+\sum_{a=1}^{2} \bar{J}_{4}^{a} \frac{d W_{a n i}}{d \bar{J}_{4}^{a}}\right]+\sum_{a=1}^{2}\left(\bar{J}_{4}^{a}\right)^{2} \frac{d^{2} W_{a n i}}{d^{2} \bar{J}_{4}^{a}}}{9 I_{3}^{2}} \\
& +\frac{U^{\prime \prime}(J) J-U^{\prime}(J)}{4 I_{3}} \\
& \frac{\partial^{2} W}{\partial I_{3} \partial J_{4}^{a}}=-\frac{1}{3} I_{3}^{-4 / 3}\left[\frac{d W_{a n i}}{d \bar{J}_{4}^{a}}+\bar{J}_{4}^{a} \frac{d^{2} W_{a n i}}{d^{2} \bar{J}_{4}^{a}}\right] \\
& \frac{\partial^{2} W}{\partial J_{4}^{a} \partial J_{4}^{a}}=I_{3}^{-2 / 3} \frac{d^{2} W_{a n i}}{d^{2} \bar{J}_{4}^{a}} \\
& U^{\prime \prime}(J)=k \\
& \frac{d^{2} W_{a n i}}{d^{2} \bar{J}_{4}^{a}}=k_{1}\left(1+2 k_{2}\left(\bar{J}_{4}^{a}-1\right)^{2}\right) \exp \left(k_{2}\left(\bar{J}_{4}^{a}-1\right)^{2}\right) H\left(J_{4}^{a}\right)
\end{aligned}
$$


où $H$ représente à nouveau la fonction de Heaviside, introduite pour prendre en compte le fait que la contribution anisotrope de la densité HGO est nulle lorsque le matériau est en compression dans la direction des fibres.

En utilisant de manière classique le principe des déplacements virtuels, les travaux virtuels $\delta W$ sont donnés par :

$$
\delta W=\delta \mathbf{u}^{\mathrm{T}} \mathbf{M} \ddot{\mathbf{u}}+\delta \mathbf{u}^{\mathrm{T}} \mathbf{A} \dot{\mathbf{u}}+\int_{V_{0}} \delta \mathbf{E}^{\mathrm{T}} \mathbf{S} d V_{0}-\delta \mathbf{u}^{\mathrm{T}} \mathbf{F}_{\text {ext }}-\delta \mathbf{u}^{\mathrm{T}} \mathbf{R}=0
$$

où $\mathbf{M}$ est la matrice de masse, $\mathbf{A}$ la matrice d'amortissement, $\ddot{\mathbf{u}}$ et $\dot{\mathbf{u}}$ l'accélération et la vitesse, $V_{0}$ le volume en configuration initiale, $\mathbf{F}_{\text {ext }}$ le vecteur des forces extérieures et $\mathbf{R}$ le vecteur des réactions de contact. L'amortissement implémenté dans FER est de type Rayleigh, c'est-à-dire que $\mathbf{A}$ est une combinaison linéaire des matrices de masse et de raideur. En substituant [30] dans [39], on obtient :

$$
\delta W=\delta \mathbf{u}^{\mathrm{T}}\left(\mathbf{M} \ddot{\mathbf{u}}+\mathbf{A} \dot{\mathbf{u}}+\int_{V_{0}}\left(\mathbf{B}_{\mathrm{L}}+\mathbf{B}_{\mathrm{NL}}(\mathbf{u})\right)^{\mathrm{T}} \mathbf{S} d V_{0}-\mathbf{F}_{\mathrm{ext}}-\mathbf{R}\right)=0
$$

On introduit le vecteur des forces internes $\mathbf{F}_{\text {int }}$ :

$$
\mathbf{F}_{\text {int }}=\int_{V_{0}}\left(\mathbf{B}_{\mathrm{L}}+\mathbf{B}_{\mathrm{NL}}(\mathbf{u})\right)^{\mathrm{T}} \mathbf{S} d V_{0}
$$

Et puisque $\delta \mathbf{u}$ est arbitraire, on déduit de [40] que :

$$
\mathbf{M u ̈}+\mathbf{A} \dot{\mathbf{u}}+\mathbf{F}_{\text {int }}-\mathbf{F}_{\text {ext }}-\mathbf{R}=\mathbf{0}
$$

Les conditions initiales associée à l'équation dynamique [42] sont données par :

$$
\dot{\mathbf{u}}=\dot{\mathbf{u}}_{0} ; \mathbf{u}=\mathbf{u}_{0}
$$

Une méthode standard pour intégrer temporellement l'équation dynamique [42] entre deux instants $t$ et $t+\Delta t$ consiste à utiliser l'algorithme explicite :

$$
\mathbf{u}^{t+\Delta t}=\Delta t^{2} \mathbf{M}^{-1}\left(\mathbf{F}_{\text {ext }}^{\mathrm{t}}-\mathbf{F}_{\text {int }}^{\mathrm{t}}+\mathbf{R}^{t+\Delta t}\right)+2 \mathbf{u}^{t}-\mathbf{u}^{t-\Delta t}
$$

Pour réaliser l'intégration numérique en distinguant les contributions sphérique et déviatorique des contraintes, il est possible d'utiliser des éléments de type Q1P0 (Scovazzi et al., 2008). Cependant, dans la version actuelle de FER, l'intégration des forces internes est effectuée de la même manière pour les parties sphérique et déviatorique en utilisant 27 points de Gauss pour les éléments hexaédriques et 1 point de Gauss pour les éléments tétraédriques.

Il est à noter que les réactions de contact $\mathbf{R}$ sont calculées avec la méthode du bipotentiel dans un système réduit ne concernant que les nœuds de contact. On aboutit alors à une équation implicite que l'on résout par un algorithme d'Uzawa, avec une 
phase de prédiction et une phase de correction. Le point-clé de la méthode est que la correction peut être calculée analytiquement en prenant en compte les trois états possibles de contact (contact adhérent, contact avec glissement et séparation). Pour plus de détails sur la méthode du bipotentiel, le lecteur intéressé pourra se référer à (De Saxcé et al., 1991 et 1998) ainsi qu'à (Feng, 1995 et 1998). L'ensemble de la démarche présentée dans ce paragraphe (formulation lagrangienne totale, méthode du bipotentiel pour séparer les non-linéarités matérielles hyperélastiques des non-linéarités locales de contact, algorithme explicite pour l'intégration temporelle) a été implémenté dans le code de calcul aux éléments finis FER (FER, 2009). La démarche est valable aussi bien en 2D qu'en 3D. Elle est illustrée en section 4 par trois exemples numériques, en quasi statique pour les deux premiers, et en dynamique explicite pour le troisième.

\section{Exemples numériques}

\subsection{Traction uniaxiale homogène}

On considère le modèle HGO dans le cadre d'un essai quasi statique de traction uniaxiale homogène (figure 2). Ce test est habituellement utilisé pour identifier les propriétés mécaniques de bandes de tissus artériels (Holzapfel, 2006 ; Balzani et al., 2006).

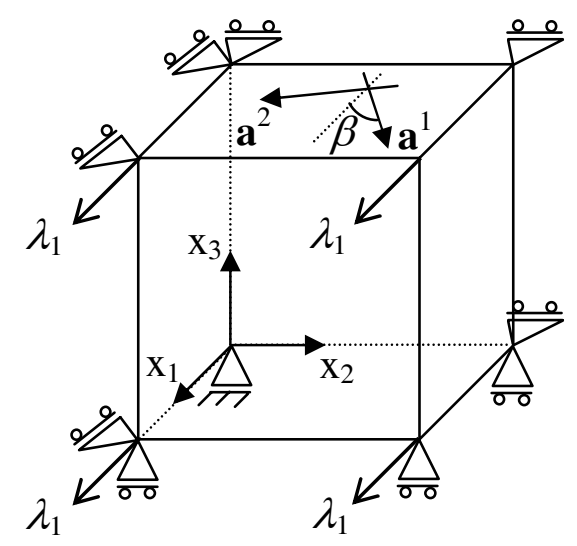

Figure 2. Essai de traction uniaxiale homogène

Comme les déformations associées à cet essai sont homogènes, il est possible de calculer analytiquement les déformations principales $\lambda_{1}, \lambda_{2}$ et $\lambda_{3}$ (Peyraut et al., 2009). Le principe consiste à résoudre les équations d'équilibre à l'aide des formules de Cardan. Ces équations s'expriment en effet sous la forme d'une équation cubique à condition d'utiliser le quatrième invariant comme paramètre. La solution dépend alors d'une discussion portant sur plusieurs critères : tissu comprimé ou en tension 
dans la direction des fibres $\left(J_{4}<1\right.$ ou $\left.J_{4} \geq 1\right)$; signe du discriminant $\Delta_{1}=t^{4} / 4$ $J_{4}{ }^{3} /\left(27 \mathrm{c}^{6}\right)$ de l'équation cubique (pour exclure par exemple les solutions non physiques associées à des racines complexes); valeur de l'angle $\beta$ par rapport à un angle critique $\beta_{\mathrm{c}}$ égal à $\operatorname{Arc} \cos (\sqrt{3} / 3) \approx 54.73$ (Guo et al., 2006). Les résultats de cette discussion se synthétisent de la manière suivante :

Si $J_{4} \geq 1$ :

$$
\begin{aligned}
& \lambda_{1}=J_{4}^{1 / 2} \cos (\theta) c^{-1}, \lambda_{2}=J_{4}^{1 / 2} \sin (\theta) s^{-1}, \cos (\theta)=2 \sqrt{3} / 3 \cos (\alpha / 3) \\
& \alpha=\operatorname{Arccos}(-3 \sqrt{3} A / 2) \\
& A=s^{2} c J_{4}^{-3 / 2}\left\{1+\frac{2 k_{1}}{c 1} s^{2}\left(J_{4}-1\right) \exp \left[k_{2}\left(J_{4}-1\right)^{2}\right]\right\}^{-1 / 2}
\end{aligned}
$$

Si $J_{4}<1$ :

si $\Delta_{1}=0$ et $\beta \geq \beta_{\mathrm{c}}$ : il existe une solution unique définie par :

$$
\lambda_{1}=\sqrt{J_{4} c^{-2} / 3}
$$

si $\Delta_{1}<0$ et $\beta \geq \beta_{\mathrm{c}}$ : il existe deux solutions distinctes $\lambda_{1 \mathrm{a}}$ et $\lambda_{1 \mathrm{~b}}$ définies par :

$$
\begin{aligned}
& \lambda_{1 a}=2 \sqrt{\frac{J_{4}}{3 c^{2}}} \cos \left(\frac{\gamma}{3}\right), \lambda_{1 b}=2 \sqrt{\frac{J_{4}}{3 c^{2}}} \cos \left(\frac{\gamma+4 \pi}{3}\right) \\
& \gamma=\operatorname{Arccos}\left[-t^{2} \sqrt{\frac{27 c^{6}}{4 J_{4}^{3}}}\right]
\end{aligned}
$$

Si $\Delta_{1}>0$ ou $\beta<\beta_{\mathrm{c}}$ : il n'existe aucune solution.

Dans le cas où $J_{4}$ est inférieur à 1 , et que la solution existe, seul le modèle néohookéen intervient. La déformation principale $\lambda_{2}$ est alors calculée en fonction de $\lambda_{1}$ par :

$$
\lambda_{2}=\lambda_{1}^{-1 / 2}
$$

Ces résultats analytiques s'interprètent de la manière suivante : pour $J_{4} \geq 1$ (fibres en extension), il existe une solution unique définie par [45]-[47] alors que le cas $J_{4}<1$ (fibres en compression) est soumis à condition. Ce cas ne peut en effet se 
présenter que si l'angle $\beta$ est supérieur à l'angle critique $\beta_{\mathrm{c}}$. La solution est alors soit unique [48], soit double [49]-[50]. Les deux situations, selon que $\beta$ soit ou non supérieur à $\beta_{\mathrm{c}}$, sont illustrées sur la figure 3 où la variation de $\lambda_{2}$ en fonction de $J_{4}$ a été représentée pour deux angles $\beta$ différents $\left(\beta=20^{\circ}\right.$ et $\left.\beta=70^{\circ}\right)$.

Situation $1\left(\beta=20^{\circ}\right)$ : dans cette situation, l'angle $\beta$ est inférieur à l'angle critique $\beta_{\mathrm{c}}$. On note que la courbe commence à $J_{4}=1$, ce qui montre bien que le cas $J_{4}<1$ ne peut avoir lieu si $\beta$ est inférieur à $\beta_{\mathrm{c}}$. La solution est en extension dans la direction des fibres $\left(J_{4} \geq 1\right)$ et correspond aux formules [45]-[47].

Situation $2\left(\beta=70^{\circ}\right)$ : Dans cette situation, l'angle $\beta$ est supérieur à l'angle critique $\beta_{\mathrm{c}}$. On note alors que la courbe $\lambda_{2}$ en fonction de $J_{4}$ comporte deux parties. La première $\left(J_{4}<1\right)$ présente la particularité de posséder deux solutions $\lambda_{2}$ pour chaque valeur de $J_{4}$. Ces deux solutions distinctes sont définies par [49] et [50]. Elles se confondent au niveau du point de rebroussement situé approximativement à $J_{4} \approx 0.85$. Ce pont correspond à la solution unique définie par [48]. La deuxième partie de la courbe $\left(J_{4} \geq 1\right)$ correspond à la solution unique définie par [45]-[47].

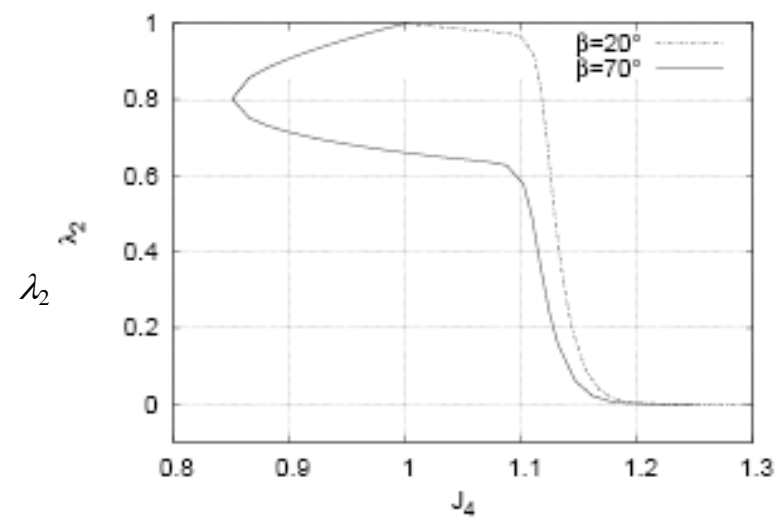

Figure 3. Courbe $\lambda_{2}-J_{4}$

La figure 4 montre l'évolution des déformations principales $\lambda_{2}$ et $\lambda_{3}$ en fonction de $\lambda_{1}$ pour un angle $\beta$ égal à $70^{\circ}$. Cette évolution a été tracée analytiquement, à l'aide des formules [45]-[50], ainsi que numériquement à l'aide de résultats obtenus avec le code de calcul FER. On observe une très bonne correspondance entre les résultats numériques et les déformations principales calculées analytiquement. On constate également que l'évolution est purement isotrope pour $\lambda_{1}$ compris entre 1 et 2.5 puisque la courbe suit manifestement une évolution donnée par l'équation [51]. Ce constat s'explique aisément si on remarque que, lorsque $\lambda_{1}$ est compris entre 1 et $2.5, \lambda_{2}$ varie dans le même temps entre 0.65 et 1 . Il suffit alors de se reporter à la courbe de la figure 3 associée à $\beta=70^{\circ}$ et de noter que, si $\lambda_{2}$ varie entre 0.65 et $1, J_{4}$ est plus petit que 1. Dans ces conditions, le modèle HGO ne prend en compte que la densité néohookéenne (section 2) et le comportement est logiquement purement isotrope. 


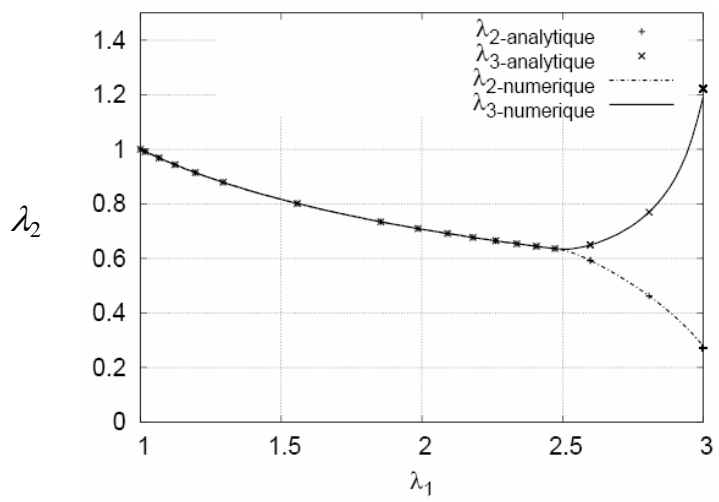

Figure 4. Courbes $\lambda_{2}$ et $\lambda_{3}$ en fonction de $\lambda_{1}-\beta=70^{\circ}$

Le cas étudié étant un essai de traction uniaxiale, la seule composante de contraintes non nulle est $\sigma_{11}$. Cette composante est représentée sur les figures 5 et 6 pour deux angles $\beta$ différents $\left(\beta=20^{\circ}\right.$ et $\left.\beta=70^{\circ}\right)$. Pour atteindre un niveau de contrainte identique, de l'ordre de $100 \mathrm{kPa}$, on remarque qu'il faut imposer une déformation environ trois fois supérieure lorsque $\beta$ est égal à $70^{\circ}$ que lorsque $\beta$ est égal à $20^{\circ}$. Cela s'explique par le fait que, plus l'angle $\beta$ formé par les fibres avec la direction de traction se rapproche de $90^{\circ}$, moins les fibres opposent de résistance à la traction imposée. Le tissu biologique est alors plus souple et, à contrainte égale, la déformation est logiquement supérieure.

La figure 7 représente un zoom sur la zone de transition anisotrope lorsque $\beta$ est égal à $70^{\circ}$. On note que cette zone est située approximativement à $\lambda_{1}$ égal à 2.5 puisqu'il y a une correspondance parfaite entre les contraintes du modèle HGO et les contraintes du modèle Néo-hookéen lorsque $\lambda_{1}$ est inférieur à 2.5. Ce constat est cohérent avec les observations déjà réalisées à l'aide des courbes reliant les déformations principales $\lambda_{1}, \lambda_{2}$ et $\lambda_{3}$ entre elles (figure 4 ).

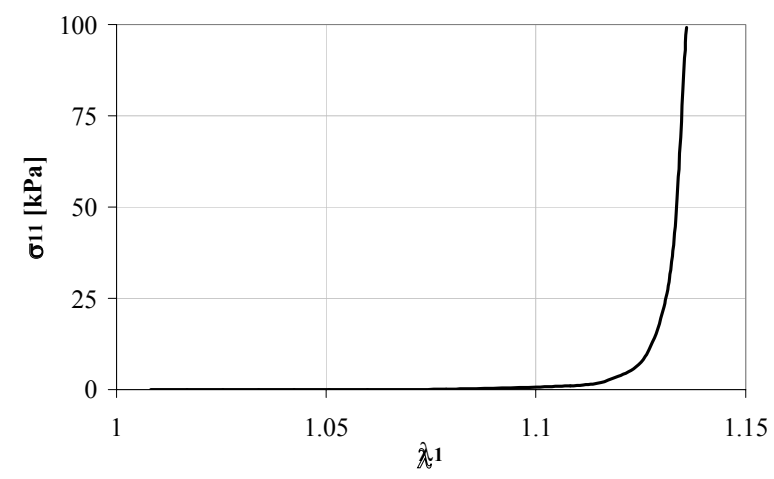

Figure 5. Contraintes de Cauchy $-\beta=20^{\circ}$ 


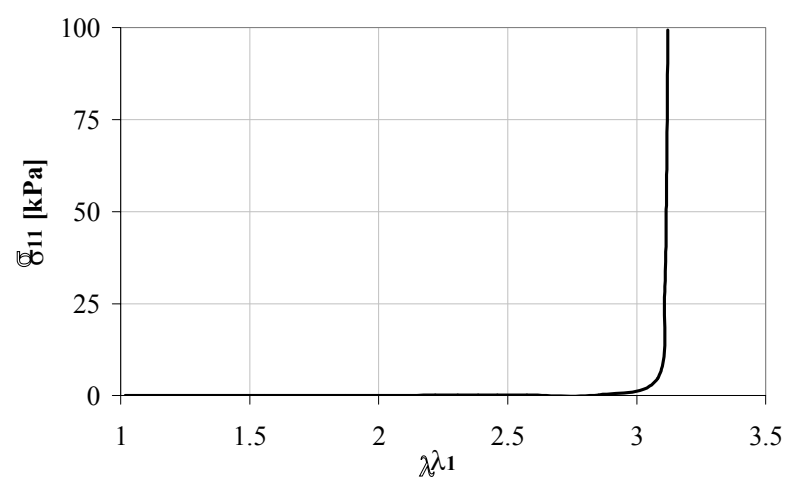

Figure 6. Contraintes de Cauchy $-\beta=70^{\circ}$

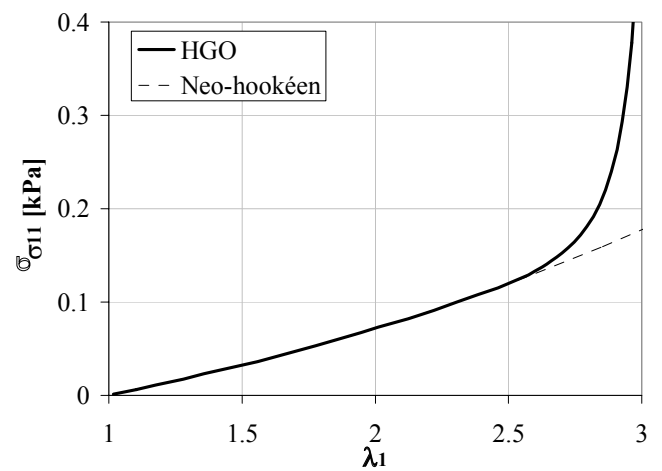

Figure 7. Contraintes de Cauchy $-\beta=70^{\circ}$-zone de transition anisotrope

\subsection{Traction uniaxiale simple}

On considère le modèle HGO dans le cadre d'un essai quasi statique de traction uniaxiale simple (figure 8). Dans ce cas, les déformations ne sont pas homogènes. De ce fait, contrairement à la section précédente, un calcul analytique n'est plus possible. La déformée numérique correspondant à la traction uniaxiale simple est présentée sur la figure 9 pour un angle $\beta$ égal à $70^{\circ}$. On note un rétrécissement de la section dans la direction 2 et un gonflement dans la direction 3 , ce qui est typique d'un comportement non isotrope. En effet, à partir d'une valeur seuil de $\lambda_{1}$, généralement annonciateur de la transition entre la compression et la tension dans la direction des fibres (cf. section 4.1), $\lambda_{3}$ augmente alors que $\lambda_{2}$ diminue. La transition s'opère lorsque la prise en compte de la densité anisotrope du modèle HGO devient effective. Ce résultat est conforme aux observations réalisées dans (Gasser et al., 2006). 


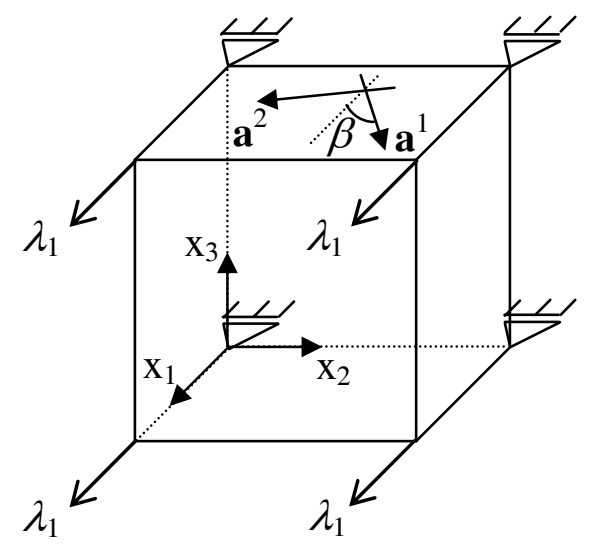

Figure 8. Essai de traction uniaxiale simple
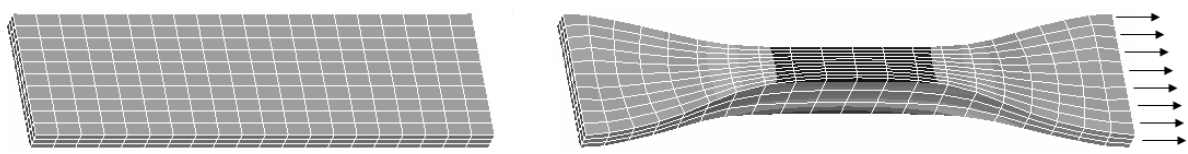

Figure 9. Test de traction uniaxiale simple - calcul numérique avec FER

Il est par ailleurs possible de quantifier la transition isotrope-anisotrope en remarquant que les déformations peuvent être considérées comme homogènes dans les régions, comme la partie centrale de l'éprouvette, qui sont suffisamment éloignées des zones d'application des conditions aux limites. Dans cette partie, la valeur de transition entre un comportement purement isotrope et un comportement orthotrope peut être calculée analytiquement en combinant l'équation [51], qui caractérise le comportement isotrope néo-hookéen, avec $J_{4}=1$, qui caractérise la transition entre un comportement purement isotrope $\left(J_{4}<1\right)$ et un comportement orthotrope $\left(J_{4}>1\right)$. On obtient alors l'équation cubique :

$$
c^{2} \lambda_{1}^{3}-\lambda_{1}+s^{2}=0
$$

Cette équation se factorise facilement puisque que $\lambda_{1}=1$ est solution évidente :

$$
\left(\lambda_{1}-1\right)\left(\lambda_{1}-\left(\sqrt{1+4 t^{2}}-1\right) / 2\right)\left(\lambda_{1}+\left(\sqrt{1+4 t^{2}}+1\right) / 2\right)=0
$$


La troisième racine étant négative, seules les deux premières sont à considérer :

$$
\lambda_{1}=1 ; \lambda_{1}=\left(\sqrt{1+4 t^{2}}-1\right) / 2
$$

Comme [51] représente la solution néo-hookéenne et que $J_{4}=1$ est l'équation d'une ellipse, les racines données par [54] représentent les abscisses des intersections entre ces deux courbes dans le plan $\left(\lambda_{1}, \lambda_{2}\right)$. Trois cas différents, correspondant à la position de l'angle $\beta$ par rapport à l'angle critique $\beta_{\mathrm{c}}$, peuvent survenir et sont illustrés sur les figures 10 à 12 . Cela met à nouveau en lumière le rôle crucial joué par l'angle critique pour l'analyse et la compréhension de la traction simple avec le modèle HGO.

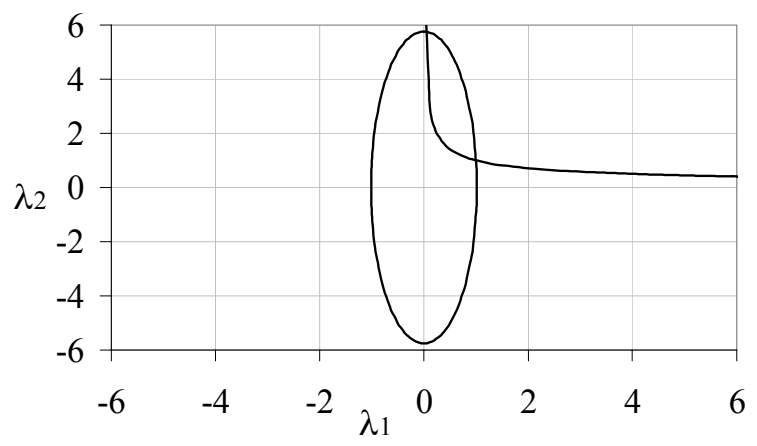

Figure 10. $\beta=10^{\circ}\left(<\beta_{\mathrm{c}}\right)$

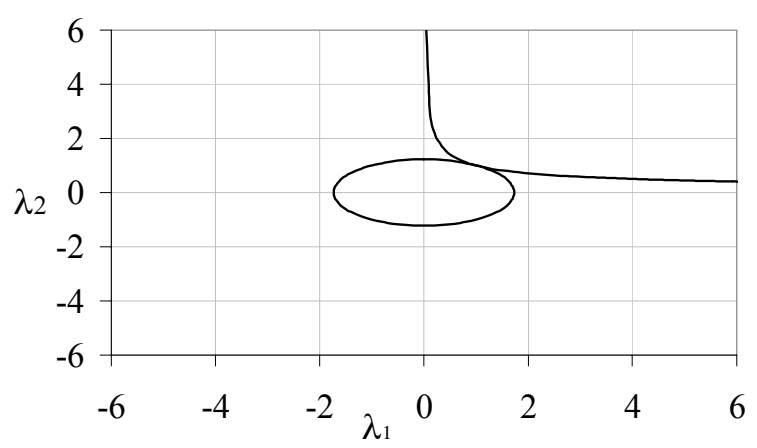

Figure 11. $\beta=\beta_{\mathrm{c}}$

Dans cette section, on se place dans le contexte de la figure 12 puisque l'angle $\beta$, égal à $70^{\circ}$, est supérieur à $\beta_{\mathrm{c}}$. Il y a donc deux intersections. La première est donnée par la solution triviale $\lambda_{1}=1$ et correspond à l'éprouvette non déformée. La seconde correspond à la transition recherchée et se calcule à partir de [54] :

$$
\lambda_{1}=\left(\sqrt{1+4 t^{2}}-1\right) / 2 \approx 2.29
$$




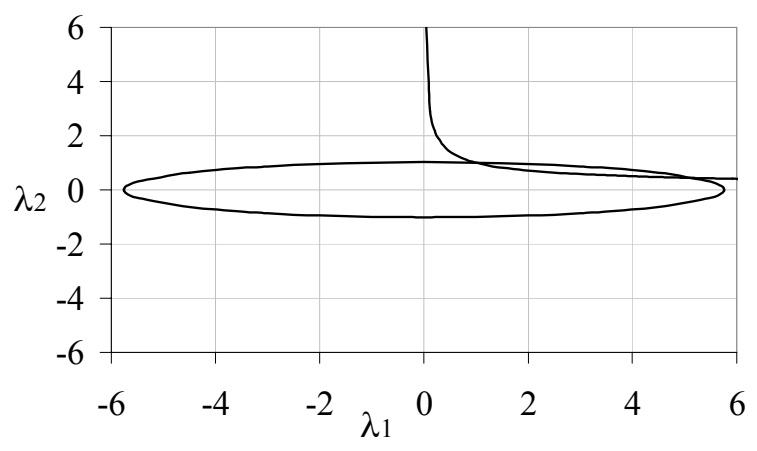

Figure 12. $\beta=80^{\circ}\left(>\beta_{\mathrm{c}}\right)$

\subsection{Application mannequin virtuel - Contact main-tissu biologique}

Dans l'exemple numérique traité dans cette section, on considère une main entrant en contact avec un échantillon de tissu biologique (figure 13). Il s'agit d'une situation que l'on rencontre usuellement dans les simulateurs de gestes chirurgicaux où les mains du praticien entrent en contact avec les organes du patient (Talbi, 2008). Le tissu est représenté par un bloc parallélépipédique dont le comportement est donné par le modèle HGO. Nous avons opté pour un comportement élastique pour la main. Ce choix n'est évidemment pas réaliste, puisque la main est constituée d'une multitude de tissus organiques au comportement hétérogène, mais cela constitue une première étape de modélisation, la main étant considérée comme plus rigide que le tissu qu'elle manipule. Le module d'Young est pris égal à $180 \mathrm{kPa}$, le coefficient de Poisson à 0.3 et la masse volumique à $1800 \mathrm{~kg} / \mathrm{m}^{3}$.

Les dimensions caractéristiques de la main sont $0.2459 \mathrm{~m}, 0.1459 \mathrm{~m}$ et $0.0820 \mathrm{~m}$ comme indiqué sur la figure 16. Celles du pavé sont représentées sur la figure 13. Le maillage de la main a été généré à partir d'une représentation triangulaire surfacique de la peau extraite du mannequin virtuel MANERCOS (Gomes et al., 1999).

Cependant, les représentations surfaciques importées de MANERCOS sont assez grossières (figure 14a) et le maillage volumique qui en résulte présente un nombre important d'aspérités et d'angles vifs (figure 14b). Ces aspérités étant susceptibles de créer des problèmes d'instabilité numérique lors du calcul de contact, nous avons procédé à un «nettoyage» préalable de la géométrie puis à une reconstruction surfacique de la main (Chamoret et al., 2009). Le résultat de cette reconstruction est présenté sur la figure 15 . Cette phase de reconstruction a été réalisée à l'aide du logiciel commercial ANSYS (ANSYS, 2007) car la version de FER que nous avons utilisée n'est pas dotée d'interface graphique pour le preprocessing, ce qui rend son emploi malaisé pour une mise en données géométrique. Le maillage obtenu comprend 5966 nœuds, 15578 éléments tétraédriques pour la main et 10947 éléments tétraédriques pour le pavé (figure 17). 
Afin de modéliser de manière réaliste la cinématique de la main d'un praticien au travail, nous avons supposé que la main impacte le bloc avec une vitesse verticale initiale $\mathrm{v}_{0}$ égale à $1 \mathrm{~m} / \mathrm{s}$ (figure 13 ). Le calcul démarre à $\mathrm{t}=0$ et le temps final est fixé à $\mathrm{t}=10^{-2} \mathrm{~s}$. La complexité de cette simulation numérique est liée à son caractère fortement non linéaire. On peut constater des discontinuités en vitesse et accélération dans le cadre de problèmes entraînant des changements brutaux d'état de contact. A cela viennent s'ajouter les non-linéarités matérielles induites par le modèle HGO. Dans ce contexte, le problème dynamique est traité par un schéma explicite mieux adapté au traitement des discontinuités dues au contact. Le choix d'un schéma explicite nécessite l'identification d'un pas de temps critique afin d'assurer la stabilité du schéma temporel (Newmark, 1959). Ce pas de temps critique est lié à la taille des éléments choisis et aux propriétés mécaniques. Une valeur critique peut être définie comme le temps nécessaire pour que l'onde de pression puisse parcourir dans un milieu élastique une longueur caractéristique égale à la taille du plus petit élément du maillage :

$$
\Delta t=\sqrt{\frac{E}{\rho}} L_{\min }
$$

où $L_{\min }$ est une longueur caractéristique du plus petit élément du maillage, $E$ est le module d'Young et $\rho$ la masse volumique. L'ordre de grandeur de $L_{\min }$ étant de $10^{-3} \mathrm{~m}$ pour le maillage de la figure 17 , la valeur critique du pas de temps est égal à $10^{-4} \mathrm{~s}$. Pour assurer la stabilité du schéma temporel avec une marge de sécurité, le pas de temps pour le calcul a été pris dix fois inférieur au pas de temps critique. Le calcul a donc été réalisé avec un pas de temps de $10^{-5} \mathrm{~s}$, sur un PC Dell muni d'un processeur de type Intel® Xeon ${ }^{\circledR}$ X5365-Quad Core $3 \mathrm{GHz}$. Il a nécessité 28 mn de temps CPU.

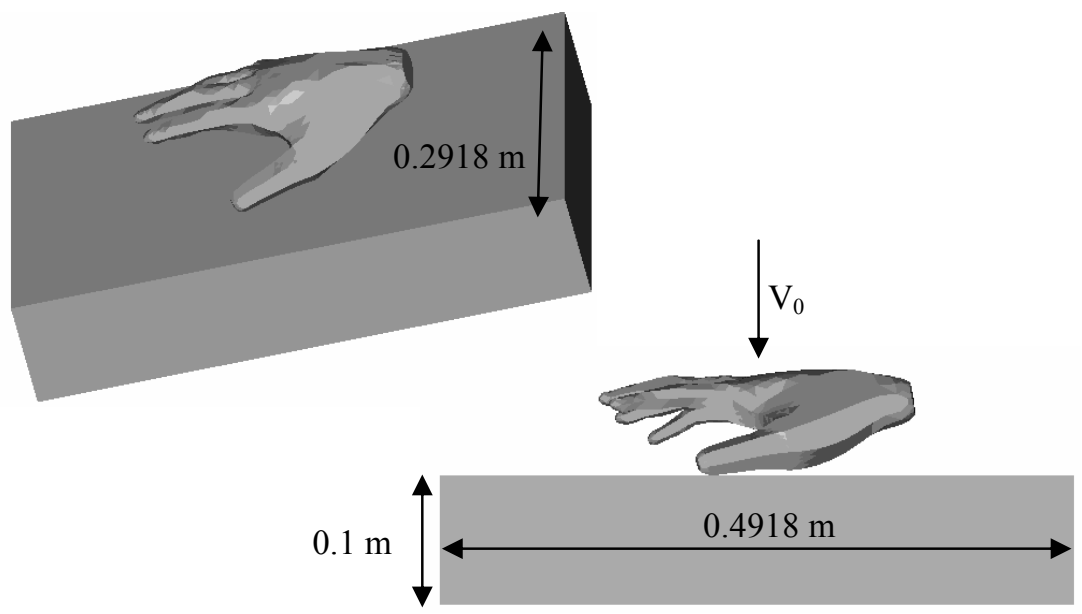

Figure 13. Main impactant un échantillon de tissu biologique 

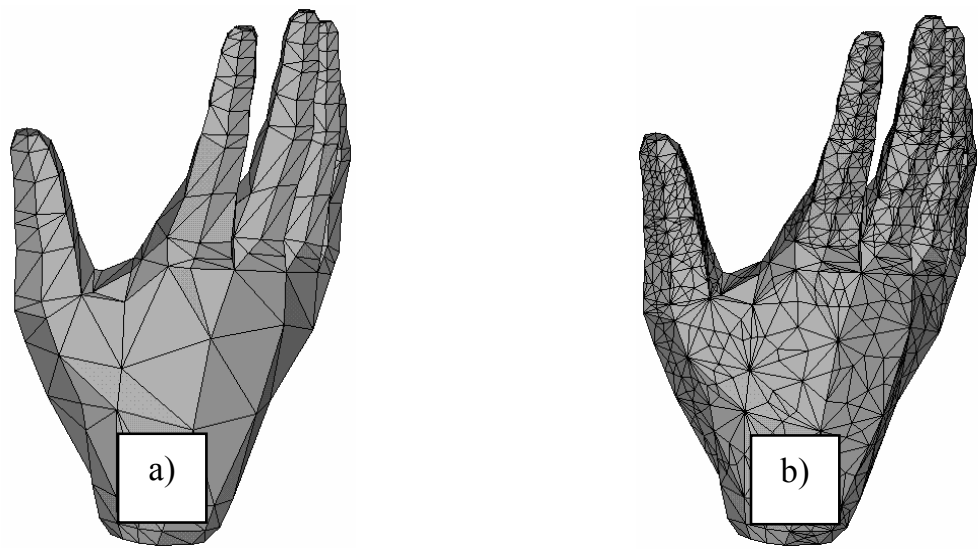

Figure 14. a) Représentation surfacique MANERCOS - b) Maillage volumique
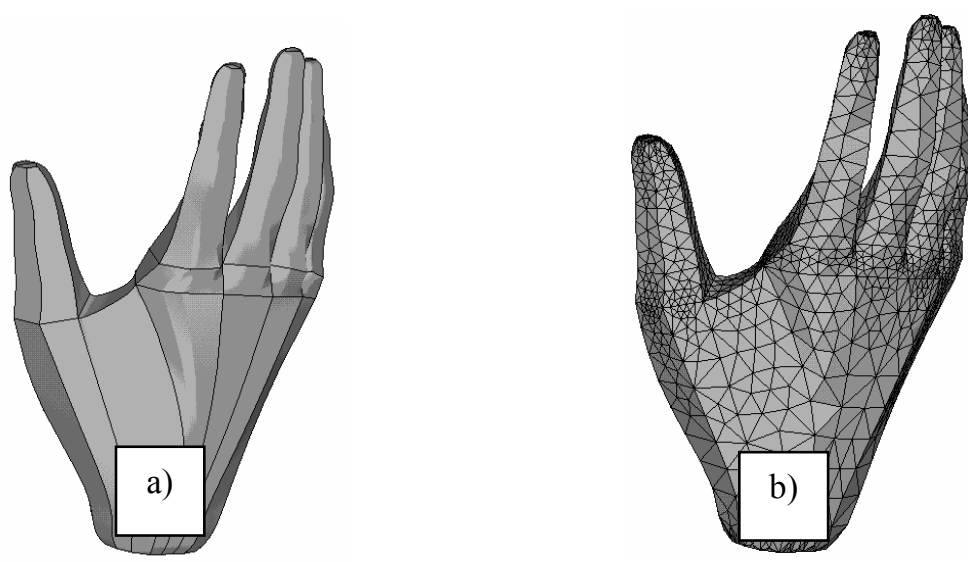

Figure 15. a) Reconstruction géométrique de la surface - b) Maillage volumique
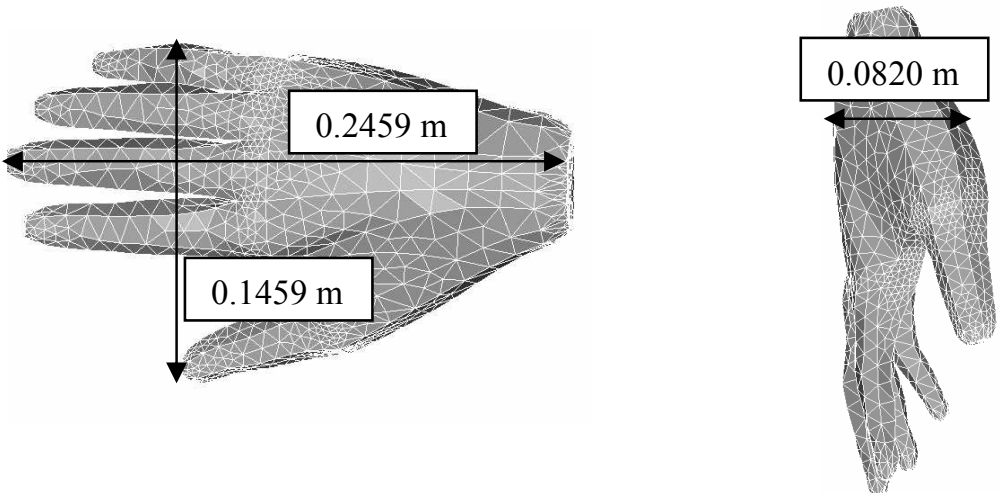

Figure 16. Dimensions de la main 


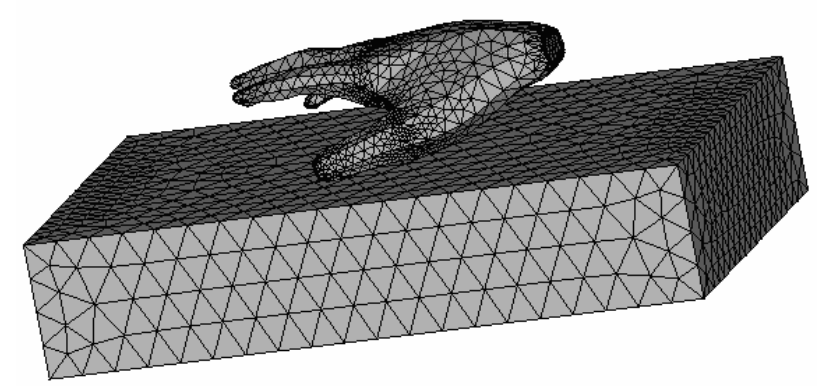

Figure 17. Maillage de la main et du pavé

La figure 18 montre l'évolution au cours du temps de la contrainte de Von Mises. Les contraintes prenant naissance dans les zones de contact entre la main et le tissu, il est possible de suivre la propagation des zones de contact au cours du temps lorsque l'appui du pouce sur le tissu devient de plus en plus important. Les déformées sont présentées avec un facteur d'amplification d'échelle égal à 2 . Comme attendu, on constate que les contraintes se propagent d'abord dans la main, dont le comportement est plus rigide que le tissu biologique, et ensuite dans le tissu.
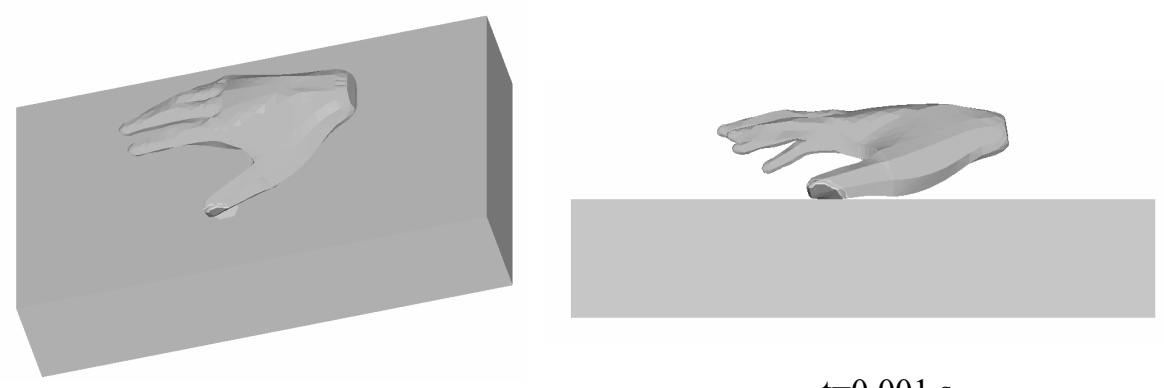

$\underline{\mathrm{t}=0.001 \mathrm{~s}}$

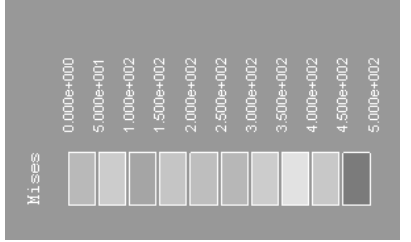



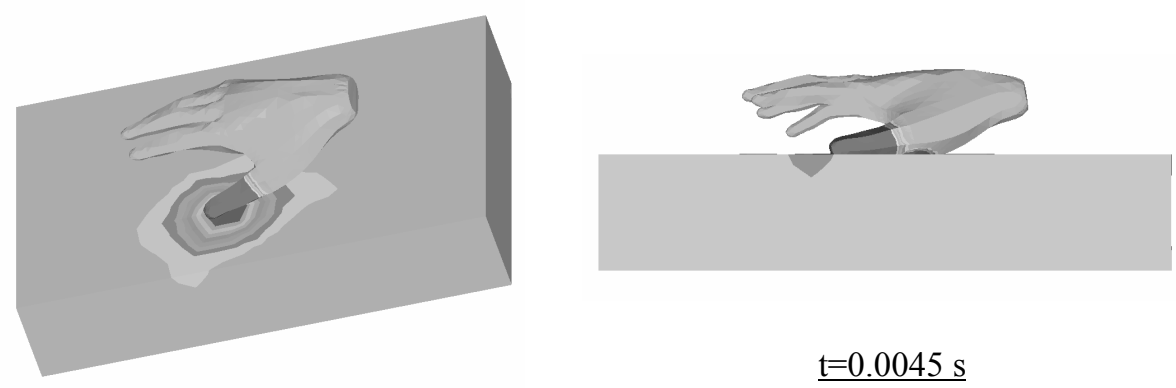

$\underline{\mathrm{t}=0.0045 \mathrm{~s}}$
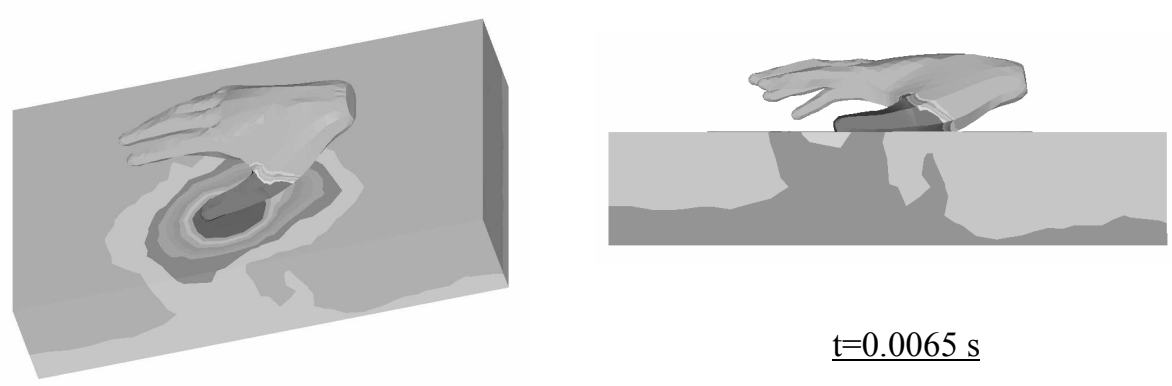

$\underline{\mathrm{t}=0.0065 \mathrm{~s}}$
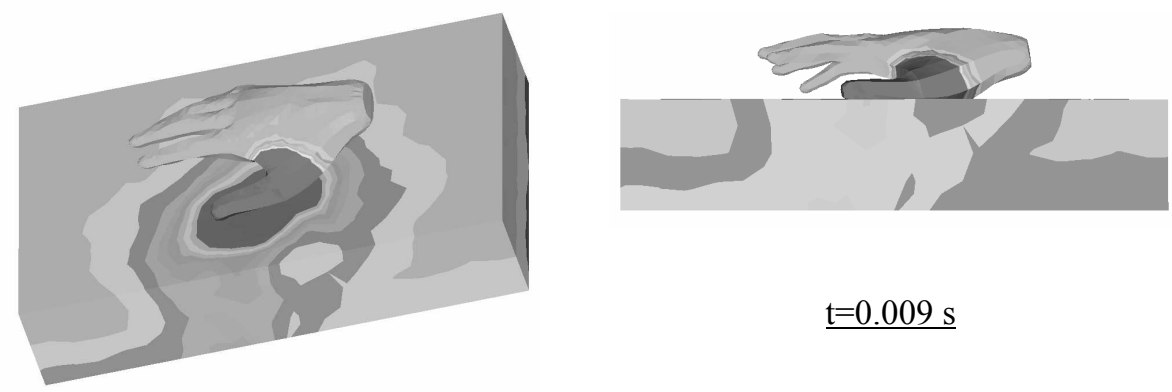

$\underline{\mathrm{t}=0.009 \mathrm{~s}}$

Figure 18. Contraintes de Von Mises à différents instants de calcul

\section{Conclusions}

On a présenté dans cet article l'implémentation éléments finis du modèle hyperélastique anisotrope HGO dans le code de calcul universitaire FER. L'implémentation a été réalisée en utilisant une approche lagrangienne totale. L'interaction avec le milieu environnant, nécessitant une gestion du contact, a été traitée par la méthode du bi-potentiel. Pour prendre en compte les phénomènes d'impact, on utilise un schéma d'intégration explicite. Trois exemples numériques 
ont été étudiés. Les deux premiers concernent des calculs quasi statiques et le troisième un calcul en dynamique explicite :

- traction uniaxiale homogène pour laquelle une solution analytique est disponible,

- traction uniaxiale simple soulignant le caractère non isotrope de la loi de comportement (rétrécissement et gonflement de la section de l'échantillon dans deux directions perpendiculaires),

- contact et impact main-tissu biologique dans le cadre d'une application avec mannequin virtuel.

Le troisième exemple est représentatif de situations rencontrées dans les simulateurs chirurgicaux de gestes opératoires. En effet, les mains du praticien interagissent avec des objets rigides, comme les instruments chirurgicaux, ou avec des tissus biologiques représentant les organes du patient virtuel. La représentation géométrique de la main a été extraite du mannequin virtuel MANERCOS. Nous avons opté pour un comportement élastique pour la main alors que le comportement des tissus manipulés est représenté par le modèle HGO. Ce choix n'est évidemment pas réaliste, puisque la main est constituée d'une multitude de tissus organiques au comportement hétérogène, mais cela constitue une première étape de modélisation, la main étant considérée comme plus rigide que le tissu qu'elle manipule. Cette première étape avait pour objectif de démontrer la faisabilité d'un calcul numérique dynamique portant sur une géométrie 3D complexe impliquant des non-linéarités locales de contact ainsi que des non-linéarités matérielles en hyperélasticité anisotrope. Il est envisagé de compléter ultérieurement le modèle 3D actuel par des éléments filaires permettant de modéliser le nombre important de coordinations articulaires et musculaires nécessaires à la préhension d'objets.

Le travail réalisé permet également d'envisager des perspectives hors applications médicales comme le prototypage virtuel. A plus longue échéance, il peut aussi ouvrir sur des challenges comme l'utilisation de lois de comportement mécanique non linéaire anisotrope en réalité virtuelle temps réel.

\section{Bibliographie}

ANSYS HTML Online Documentation, Release 11.0 Documentation for ANSYS, 2007.

Balzani D., Neff P., Schröder J., Holzapfel G.A, “A polyconvex framework for soft biological tissues. Adjustment to experimental data”, International Journal of Solids and Structures, $\mathrm{n}^{\circ} 43,2006$, p. 6052-6070.

Bathe K.J., Finite element procedures in engineering analysis, Englewood Cliffs, NJ: Prentice-Hall, 1982.

Chamoret D., Peyraut F., Gomes S., Mahdjoub M., Chevriau S., Feng Z.-Q., « Du mannequin numérique au calcul éléments finis - application à la modélisation en biomécanique », $11^{e}$ Colloque National AIP PRIMECA de La Plagne, 22-24 avril 2009. 
De Saxcé G., Feng Z.-Q., "New inequality and functional for contact with friction: the implicit standard material approach", Mech. Struct. Mach., n 19, 1991, p. 301-325.

De Saxcé G., Feng Z.-Q., "The bi-potential method: a constructive approach to design the complete contact law with friction and improved numerical algorithms", Math. Comput. Model, Special issue: Recent Advances in Contact Mechanics, $n^{\circ} 28$ (4-8), 1998, p. 225-245.

Feng Z.-Q., "2D or 3D frictional contact algorithms and applications in a large deformation context", Commun. Numer. Methods Eng., n 11, 1995, p. 409-416.

Feng Z.-Q., "Some test examples of 2D and 3D contact problems involving coulomb friction and large slip", Math. Comput. Model. Special issue: Recent Advances in Contact Mechanics, $\mathrm{n}^{\circ} 28$ (4-8), 1998, p. 469-477.

Feng Z.-Q., Cros J.-M., "FER/SUBDomain an integrated environment for finite element analysis using object-oriented approach", Mathematical Modelling and Numerical Analysis, vol. 36, $\mathrm{n}^{\circ}$ 5, 2002, p. 773-781.

FER Online Documentation, http://lmee.univ-evry.fr/ feng/FerSystem.html, 2009.

Fung Y.C., Fronek K., Patitucci P., "Pseudoelasticity of arteries and the choice of its mathematical expression", Am. J. Physiol., n 237, 1979, p. 620-631.

Gasser T.C., Ogden R.W., Holzapfel G.A., "Hyperelastic modelling of arterial layers with distributed collagen fibre orientations", J. R. Soc. Interface, n ${ }^{\circ} 3,2006$, p. 15-35.

Gomes S., Sagot J.-C., Koukam A., Leroy N., "MANERCOS, a new tool providing ergonomics in a concurrent engineering design life cycle", $4^{\text {th }}$ Annual Scientific Conference on Web Technology, New Media, Communications and Telematics - Theory, Methods, Tools and Applications, EUROMEDIA 99, Munich, 25-28 April 1999, p. 237-24.

Guo Z.Y., Peng X.Q., Moran B., “A composites-based hyperelastic constitutive model for soft tissue with application to the human annulus fibrosus" J. Mech. Phys. Solids, ${ }^{\circ} 54$, 2006, p. 1952-1971.

Holzapfel G.A., Gasser T.C., Ogden R.W., "A new constitutive framework for arterial wall mechanics and a comparative study of material models", Journal of Elasticity, $\mathrm{n}^{\circ} 61$, 2000, p. $1-48$.

Holzapfel G.A., "Determination of material models for arterial walls from uniaxial extension tests and histological structure", Journal of Theoretical Biology, 238, 2006, p. 290-302.

Newmark N.M., "A method of computation for structural dynamics", Journal of the Engineering Mechanics Division, ASCE, 85, 1959, p. 67-94.

Peyraut F., Renaud C., Labed N., Feng Z.-Q., "Modélisation de tissus biologiques en hyperélasticité anisotrope - Etude théorique et approche éléments finis", Comptes Rendus Mécanique, vol. 337, n², 2009, p. 101-106.

Schröder J., Neff P., Balzani D., “A variational approach for materially stable anisotropic hyperelasticity", International Journal of Solids and Structures, $\mathrm{n}^{\circ} 42$, 2005, p. 4352 4371. 
Scovazzi G., Love E., Shashkov M.J., "Multi-scale Lagrangian shock hydrodynamics on Q1/P0 finite elements: Theoretical framework and two-dimensional computations", Computer methods in applied mechanics and engineering, $\mathrm{n}^{\circ} 197,2008$, p. 1056-1079.

Spencer A.J.M., Isotropic polynomial invariants and tensor functions, Boehler, J.P. (Ed.), Applications of Tensor Functions in Solids Mechanics, CISM Course No. 282. Springer Verlag, 1987.

Talbi N., Résolution du contact frottant entre objets déformables en temps réel et avec retour haptique, Thèse de Doctorat, Université d'Evry Val d'Essonne, 2008.

Weiss J.A., Maker B.N., Govindjee S., "Finite element implementation of incompressible, transversely isotropic hyperelasticity", Computer methods in applied mechanics and engineering, $\mathrm{n}^{\circ} 135,1996$, p. 107-128.

Received: 26 March 2009

Accepted: 21 October 2009 